\title{
Modified Iliac Crest Reconstruction with Bone Cement for Reduction of Donor Site Pain and Morbidity after Open Wedge High Tibial Osteotomy: A Prospective Study
}

\author{
Jong Seong Lee, $\mathrm{MD}^{1}$, Yong Jee Park, $\mathrm{MD}^{2}$, Lih Wang, $\mathrm{MD}^{3}$, Yong Suk Chang, $\mathrm{MD}^{1}$, Gautam M. Shetty, $\mathrm{MD}^{4}$, \\ and Kyung Wook Nha, $\mathrm{MD}^{1}$ \\ ${ }^{1}$ Department of Orthopaedic Surgery, Inje University Ilsan Paik Hospital, Goyang; ${ }^{2}$ Department of Orthopaedic Surgery, Bang Haw Hospital, Seoul; ${ }^{3}$ Department of \\ Orthopaedic Surgery, Dong-A University Hospital, Busan, Korea; ${ }^{4}$ Asian Orthopaedic Institute, Asian Heart Institute and Research Centre, Mumbai, India
}

Purpose: This study was to determine the efficacy of iliac crest reconstruction using bone cement in reducing pain and morbidity at the donor site in patients undergoing open wedge high tibial osteotomy (OWHTO) with tricortical iliac crest autologous graft.

Materials and Methods: Thirty-three patients who underwent iliac crest reconstruction using polymethyl methacrylate (PMMA) bone cement (group A) and thirty patients who had no iliac crest reconstruction (group B) were enrolled in this study. All patients were evaluated for pain and functional disability related to graft harvesting using the pain and functional visual analogue scale (VAS) score during hospital stay and at 6 weeks, 3 months, and 6 months postoperatively.

Results: There was significant difference between the two groups in terms of pain and function. The pain VAS score was significantly lower in group A than group B during the first 2 weeks postoperatively $(\mathrm{p}=0.04)$ and the functional VAS score was also significantly lower in group A during the first 2 weeks postoperatively $(\mathrm{p}<0.001)$ in terms of breathing, sitting up from the supine position, and standing up with crutches from the sitting position. Conclusions: Iliac crest donor site reconstruction using PMMA bone cement in patients undergoing OWHTO significantly decreased pain and improved function during the first 2 weeks postoperatively when compared to patients who underwent OWHTO without iliac crest reconstruction.

Keywords: Tibia, Osteotomy, Bone transplantation, Polymethyl methacrylate

\section{Introduction}

Autologous bone graft from the iliac crest is commonly used for the spine fusion, nonunion of fracture and opening wedge high tibial osteotomy (OWHTO). However, donor site morbidity and the requirement for a second operation field have been major

Received July 27, 2015; Revised (1st) October 19, 2015;

(2nd) September 26, 2016; Accepted October 14, 2016

Correspondence to: Kyung Wook Nha, MD

Department of Orthopaedic Surgery, Inje University Ilsan Paik Hospital, 170 Juhwa-ro, Ilsanseo-gu, Goyang 10380, Korea

Tel: +82-31-910-7312, Fax: +82-31-910-7319

E-mail:kwnhamj@hotmail.com

This is an Open Access article distributed under the terms of the Creative Commons Attribution Non-Commercial License (http://creativecommons.org/licenses/by-nc/4.0/) which permits unrestricted non-commercial use, distribution, and reproduction in any medium, provided the original work is properly cited. problems. In addition, acute severe pain at the iliac site is one of the major complications ${ }^{1)}$ although most donor site pain usually resolves with time, and a small number of patients experience persisting pain. Early postoperative donor site pain causes reduced ambulation leading to prolonged hospital stay and delay in resumption of work and other activities ${ }^{2}$. Despite these disadvantages, iliac crest autograft remains the gold standard for numerous reasons including cost-effectiveness, immune compatibility, and reduced infection risk. No substitute can replace its combined osteoinductive, osteoconductive potential in promoting bone union. In countries where cost is an additional significant constraint, autograft remains a reliable, attractive, and easily accessible option. In our previous study, we used the iliac tricortical bone graft for supporting the medial tibial defect in $\mathrm{OWHTO}^{3)}$.

Several techniques have been suggested to reduce the incidence or severity of donor site pain using calcium phosphate cement, 
a tricalcium phosphate bone filler, or a restorable mesh in spine surgery ${ }^{2,4,5)}$. In this study, we applied a new technique for bone cement filling in the large iliac tricortical bone defect in OWHTO.

The purpose of this study was to evaluate the efficacy of the modified iliac crest bone cement reconstruction as a method for reducing donor-site pain and morbidity in patients undergoing OWHTO using tricortical iliac crest autograft compared to those who did not undergo iliac crest reconstruction in OWHTO. We hypothesized that the modified iliac crest bone reconstruction using bone cement in patients undergoing OWHTO would be effective in reducing postoperative pain and morbidity at the donor site.

\section{Materials and Methods}

All patients who were scheduled to undergo OWHTO for medial knee osteoarthritis at a single institution between 2010 and 2013 were eligible for enrollment in this prospective randomized controlled clinical trial study. The inclusion criteria were patients undergoing OWHTO with iliac crest tricortical autograft under general anesthesia for medial osteoarthritis or osteonecrosis, who had provided informed consent for their participation. The exclusion criteria were inability to tolerate general anesthesia for the procedure, discontinuation of intravenous patient-controlled analgesia (PCA) postoperatively, preoperative deformity of varus 15 degrees, previous operation or refusal to participation. The study was approved by the Institutional Review Board of the hospital.

\section{Study Design}

A total of 81 patients underwent the procedure during the study period. Based on the exclusion criteria, 12 patients were excluded from the study: 3 due to refusal of participation, 2 due to inability to receive general anesthesia, and 7 due to discontinuation of intravenous PCA postoperatively. Therefore, 69 patients who underwent OWHTO were enrolled in this study. The patients were randomly allocated to a group with polymethyl methacrylate (PMMA) or a group without PMMA based on a random number table. Six patients dropped out of the study due to followup loss. Accordingly, the final analysis included data from 33 patients in the group with PMMA (group A) and 30 patients in the group without PMMA (group B) (Fig. 1). The surgery was performed by a single senior surgeon and the same postoperative rehabilitation protocol was used in all patients. All of the patients were assessed preoperatively for demographic data including age, gender, body mass index (BMI), and preoperative status.

\section{Operative Technique}

Both groups underwent the same procedure: OWHTO with tricortical iliac crest autograft fixed with AO T-plate and nonlocking system (Synthes, Oberdorf, Switzerland) ${ }^{3)}$. Ttricortical

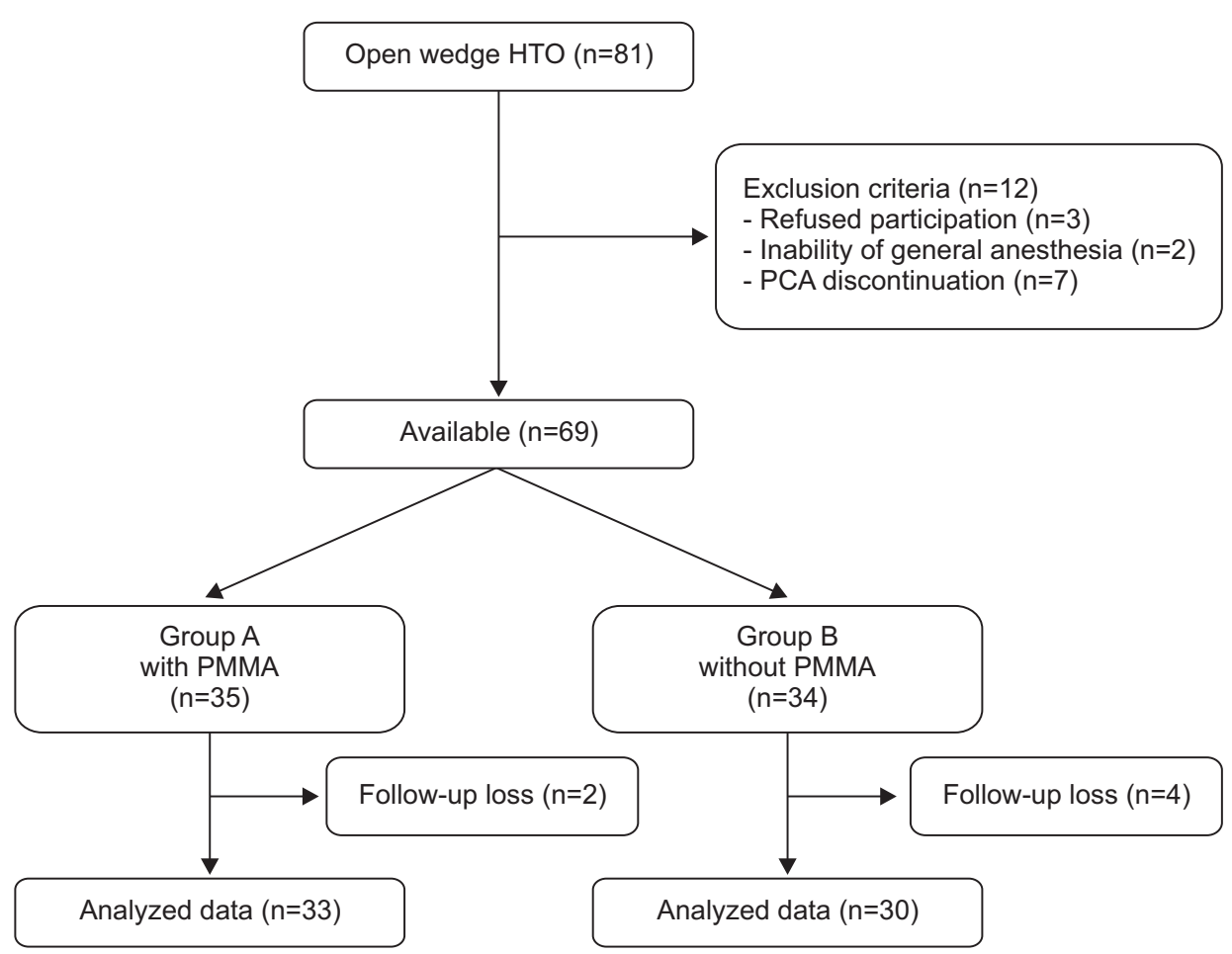

Fig. 1. Flow diagram of the study. HTO: high tibial osteotomy, PCA: patient-controlled analgesia, PMMA: polymethyl methacrylate. 
iliac crest graft was harvested in both groups using the same technique. The contralateral anterior iliac crest was approached through a $4 \mathrm{~cm}$ incision made over the anterior iliac crest and the periosteum was sharply incised and reflected. The graft size was first delineated on the bone using electrocautery: the average graft size was $30 \mathrm{~mm}$ in length, $10-15 \mathrm{~mm}$ in width, and $40 \mathrm{~mm}$ in depth. The tricortical iliac crest graft consisting of the anterior crest and the inner and outer iliac cortices was then harvested using a sharp osteotome. The only procedural difference between the groups was that the iliac crest graft site was reconstructed using PMMA bone cement in group A whereas no such reconstruction was performed in group B. In group A, two grooves were formed by a bone burr into each wall of the iliac crest defect to anchor the cement block within the defect thereby preventing

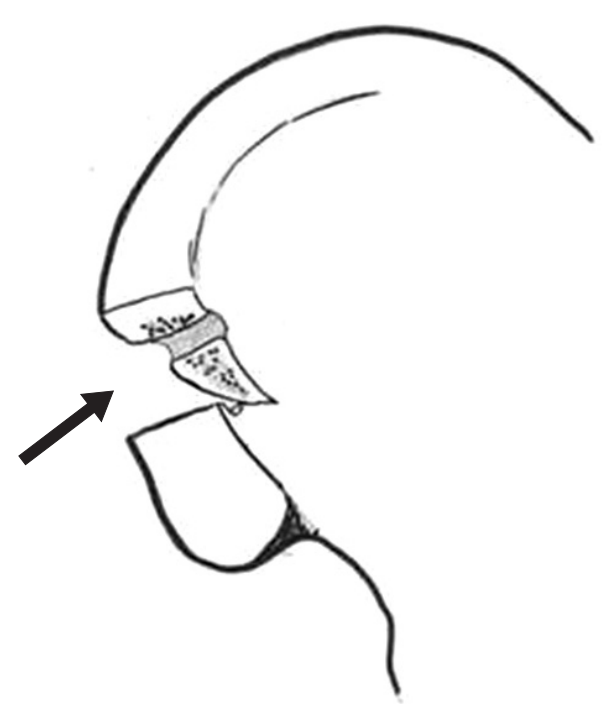

Fig. 2. Donor site preparation prior to application of bone cement involved bone burr cutting of grooves (arrows) into each wall of the defect. dislodgement of cement from the donor site postoperatively (Figs. 2 and 3$)$.

\section{Postoperative Management}

Both groups received the same pain-control regimen, which involved the use of an intravenous PCA system for 3 days, intravenous tramadol $(50 \mathrm{mg}$ ) every 8 hours for 3 days, and oral aceclofenac (100 mg) every 12 hours until discharge. Both groups underwent the same postoperative rehabilitation regimen, which included isometric quadriceps exercises, active ankle exercises, and straight leg raises on the first postoperative day; knee flexion from $0^{\circ}$ to $100^{\circ} 3$ days a week at 1 week postoperatively; toe touch weight bearing at 1 week postoperatively; and partial weight bearing at 2 weeks postoperatively. All patients were discharged at 2 weeks postoperatively and full weight bearing was allowed after 4 weeks. No cast or brace was used postoperatively.

\section{Assessment}

Postoperative pain at rest was assessed using the visual analogue scale (VAS) score (pain VAS) in both groups. Functional disability was assessed according to the degree of pain felt while performing 3 daily routine activities (functional VAS): 1) breathing, 2) sitting up from the supine position, and 3) standing up with crutches from the seated position. The pain VAS score was recorded by an orthopedic resident every day till discharge and at 6 weeks, 3 months, and 6 months postoperatively whereas the functional VAS score was recorded only at the time of discharge (or 2 weeks postoperatively).

\section{Statistical Analysis}

To achieve a power of 0.8 and an alpha value of 0.05 , the required sample size was 50 patients in total ( 25 patients for each
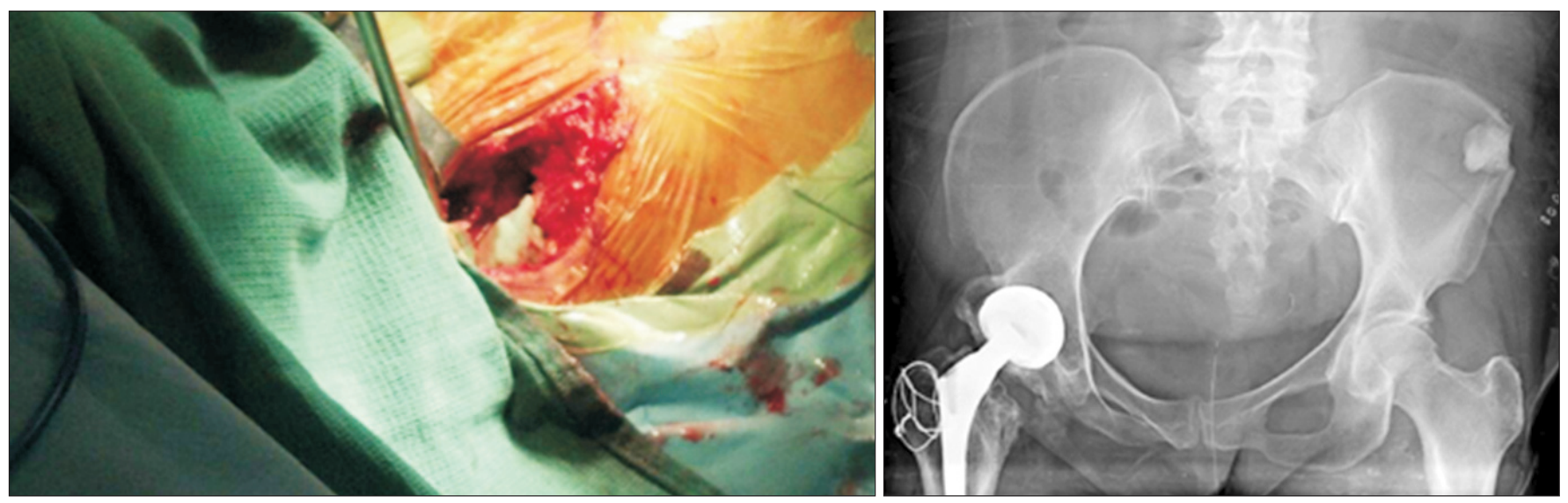

Fig. 3. Clinical photograph and postoperative pelvic radiograph showing the iliac crest after bone cementing. 
group). When a 20 percent drop-out rate was assumed in the calculation, the expected sample size was at least 30 patients in each group. The calculation was based on the pain score at rebound pain as described in a previous publication ${ }^{6}$. Both groups were compared with regard to preoperative demographic parameters and the pain VAS/functional VAS scores using the Student $t$-test. All statistical analyses were performed using the SPSS ver. 18.0 (SPSS Inc., Chicago, IL, USA). A p $<0.05$ was considered to be statistically significant.

\section{Results}

There were no significant differences in demographic parameters (gender, age, BMI, or preoperative status) between the two groups (Table 1).

The pain VAS score was significantly lower in group A when

Table 1. Comparative Results of Demographic Characteristics between Group A and Group B

\begin{tabular}{lccc}
\hline \multicolumn{1}{c}{ Characteristic } & $\begin{array}{c}\text { Group A } \\
\text { (cement) }\end{array}$ & $\begin{array}{c}\text { Group B } \\
\text { (no cement) }\end{array}$ & p-value \\
\hline No. of patients & 33 & 30 & \\
Age (yr) & $52.36(43-63)$ & $54.36(43-64)$ & 0.61 \\
Sex (M:F) & $13: 20$ & $11: 19$ & 0.83 \\
Height (cm) & $160.42 \pm 8.43$ & $160.10 \pm 8.69$ & 0.88 \\
Weight $(\mathrm{kg})$ & $69.75 \pm 13.26$ & $67.95 \pm 9.57$ & 0.54 \\
Body mass index $\left(\mathrm{kg} / \mathrm{m}^{2}\right)$ & $26.96 \pm 3.76$ & $26.49 \pm 2.91$ & 0.57 \\
Mechanical axis $\left(^{\circ}\right)$ & $5.82 \pm 3.53$ & $5.63 \pm 4.00$ & 0.83 \\
\hline
\end{tabular}

Values are presented as median \pm standard deviation (range).

${ }^{a)}$ Statistically significant, $\mathrm{p}<0.05$.

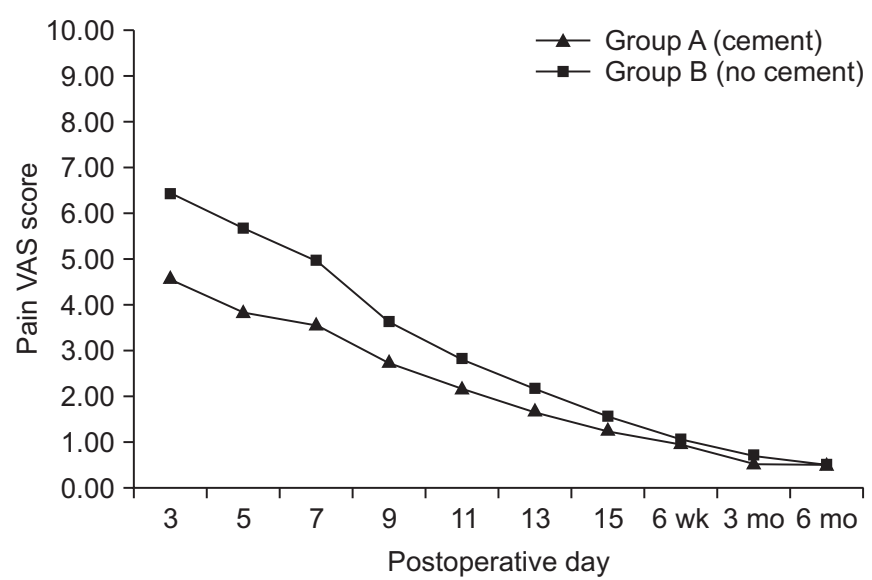

$1 \mathrm{wk}(p<0.001), 2 \mathrm{wk}(p=0.04), 6 \mathrm{wk}(p=0.99), 3 \mathrm{mo}(p=0.23)$, and $6 \mathrm{mo}(p=0.92)$

Fig. 4. Comparison of pain visual analogue scale (VAS) scores between two study groups for 6 postoperative months. compared to group B during the first 2 weeks postoperatively (Fig. 4). The pain VAS score was significantly lower in group A at 1 week $(p<0.001)$ and 2 weeks $(p=0.04)$ postoperatively whereas it showed no significant difference between the two groups at 6 weeks $(p=0.99), 3$ months $(p=0.23)$, and 6 months $(p=0.92)$ postoperatively (Fig. 4). The functional VAS score was significantly lower $(\mathrm{p}<0.001)$ in group A when compared to group B at discharge (or 2 weeks postoperatively) for all 3 daily activities of breathing, sitting up from the supine position, and standing up with crutches from the sitting position (Fig. 5).

There were two complications in one case in group A which involved loosening and displacement of the PMMA bone cement used to reconstruct the iliac crest (Fig. 6). And there were two cases of hematoma. However, there were no cases of iliac crest fracture or delayed wound healing at the donor site. The complications in group B (no PMMA) were three pelvic bone fractures

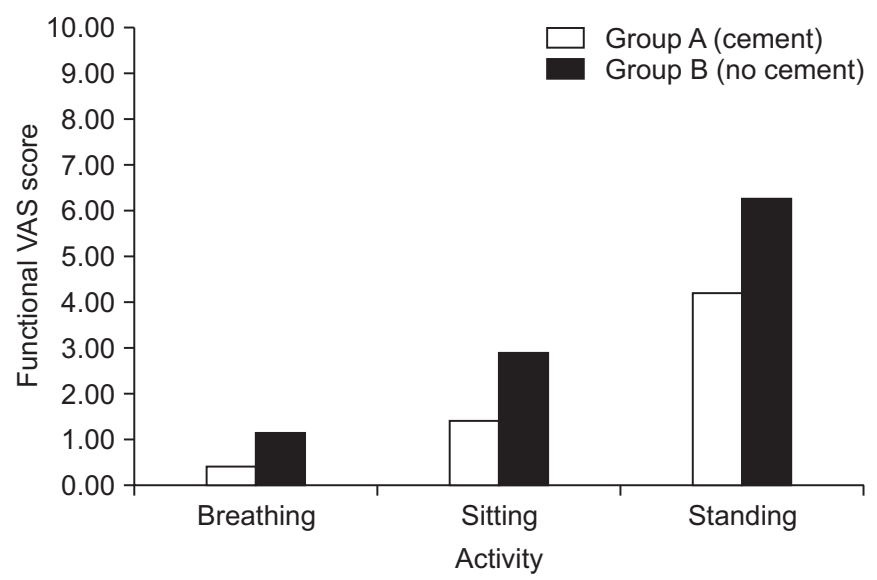

Fig. 5. Comparison of functional visual analogue scale (VAS) scores between two groups for 2 postoperative weeks. The functional VAS score was significantly lower $(\mathrm{p}<0.001)$ in group A than in group $B$.

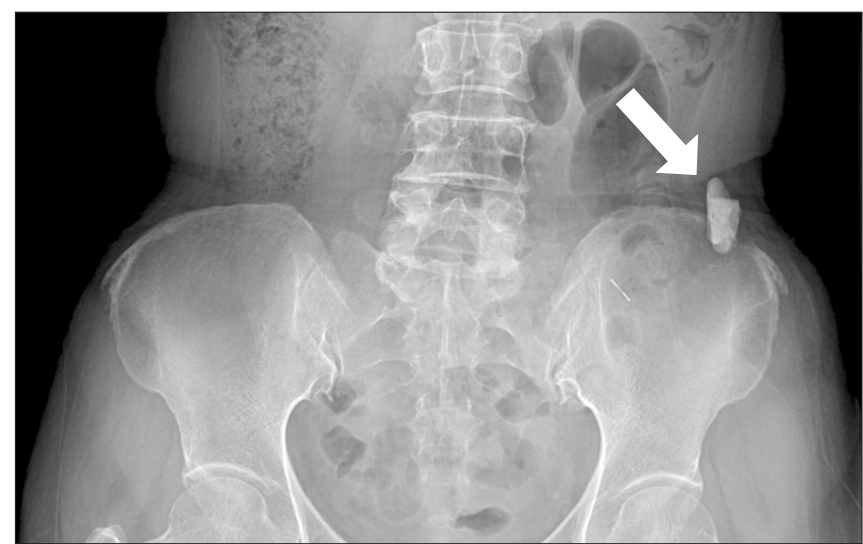

Fig. 6. Postoperative pelvic radiograph showing dislodgement of the cement wedge (arrow). 
(linear type), two hematomas, and one infection.

Complications were treated by surgical removal in one case and drainage and antibiotics in the other two cases in group A. Patients were discharged by the end of the 2 nd postoperative week.

\section{Discussion}

Persistent pain at the iliac crest donor site, reported extensively in patients undergoing spine surgery ${ }^{1,2,4,7,8)}$, may also occur in patients undergoing OWHTO, causing postoperative morbidity ${ }^{9)}$. Reconstruction of the iliac crest donor site can alleviate donor site pain and morbidity by reducing the formation of adhesive scars and preventing irritation caused by edges of the donor site ${ }^{10)}$. Our prospective study showed that the modified reconstruction of the iliac crest donor site using PMMA bone cement significantly reduced pain and functional disability in patients undergoing open OWHTO for at least up to 2 weeks postoperatively.

The results of our study are similar to those of Resnick ${ }^{4)}$ who reported significant pain alleviation at the iliac graft donor site at 6 weeks and 3 months postoperatively after reconstruction with tricalcium phosphate bone substitute. However, in comparison to the study by Resnick ${ }^{4}$, there was no significant difference in pain reduction between the two groups after 2 postoperative weeks in our patients. This discrepancy in the results could be attributable to two reasons: the size of the graft harvested and the material used to reconstruct the iliac bone defect (bone cement versus tricalcium phosphate). The size of iliac crest graft harvested for OWHTO in our study $(30 \times 15 \times 40 \mathrm{~mm})$ was much larger that harvested for cervical spine surgery in the Resnick's study (range, 12 to $16 \mathrm{~mm})^{4)}$. Bone substitutes such as tricalcium phosphate may not be available at all medical centers, and add considerably to the cost of procedure compared to the PMMA bone cement.

The mechanism of the pain is unclear, but it has been presumed to be trauma to the periosteum or muscle. Several methods have been suggested to reduce the donor site pain such as rounding of iliac bony edges ${ }^{11)}$, insertion of a resorbable mesh ${ }^{5)}$, and a local injection of morphine ${ }^{12)}$.

In our study, the mechanism of pain relief after iliac crest reconstruction with cement may be related to 1) prevention of micromotion at the defect site, 2) initial support by cement fixation at the bone defect site, or 3) protection of the graft site against adhesive scar formation.

The complications encountered in patients who underwent iliac crest reconstruction with PMMA bone cement (group A) included loosening and displacement of the cement block in one patient and hematoma without pelvic bone fracture in two patients. For cement loosening, surgical removal was carried out and recovery was uneventful. The loosening and displacement of the cement block was probably due to inadequate anchoring of cement block at the donor site and improper tightness of the soft tissue after repair. The modified iliac crest reconstruction creates two grooves through electric burring in either wall of the donor site in order to firmly anchor the cement block within the defect. The cement technique may have prevented pelvic bone fracture due to initial support at the bone donor site in group A.

PMMA bone cement is relatively low cost, easily available, and effective. There are several options to reconstruct the iliac crest graft donor site. These include using bone graft substitutes ${ }^{4,13)}$, calcium phosphate cement ${ }^{2}$, a ceramic spacer ${ }^{14)}$, and iliac crest allograft ${ }^{10)}$. However, all these options result in a higher cost to the patient compared to the easily available PMMA bone cement. Furthermore, using allograft or any bone substitute at the donor site may cause issues related to union and integration.

In this study, we maintained treatment consistency to the best possible extent in both groups. The graft harvesting technique, immediate postoperative pain management protocol, and postoperative rehabilitation regiment were the same in all patients and the questionnaire used was devised by a single senior surgeon. Nonetheless, there are several possible limitations of this study. First, the sample size was relatively small; a bigger sample size should have been used. In addition, the study population was predominantly female (41 of 63 patients), although women are more susceptible to knee osteoarthritis for some unknown reasons. Finally, the study was conducted by only one surgeon at one institution; a multi-center study involving more surgeons could strengthen the validity of the results.

\section{Conclusions}

The modified iliac crest donor site reconstruction using PMMA bone cement in patients undergoing OWHTO significantly decreased pain and improved function during the first 2 weeks postoperatively when compared to those who did not undergo iliac crest reconstruction.

\section{Conflict of Interest}

No potential conflict of interest relevant to this article was reported. 


\section{References}

1. Chau AM, Xu LL, van der Rijt R, Wong JH, Gragnaniello C, Stanford RE, Mobbs RJ. Reconstruction versus no reconstruction of iliac crest defects following harvest for spinal fusion: a systematic review: a review. J Neurosurg Spine. 2012; 16:565-72.

2. Dusseldorp JR, Mobbs RJ. Iliac crest reconstruction to reduce donor-site morbidity: technical note. Eur Spine J. 2009; 18:1386-90.

3. Chae DJ, Shetty GM, Lee DB, Choi HW, Han SB, Nha KW. Tibial slope and patellar height after opening wedge high tibia osteotomy using autologous tricortical iliac bone graft. Knee. 2008;15:128-33.

4. Resnick DK. Reconstruction of anterior iliac crest after bone graft harvest decreases pain: a randomized, controlled clinical trial. Neurosurgery. 2005;57:526-9.

5. Wang MY, Levi AD, Shah S, Green BA. Polylactic acid mesh reconstruction of the anterior iliac crest after bone harvesting reduces early postoperative pain after anterior cervical fusion surgery. Neurosurgery. 2002;51:413-6.

6. Koh IJ, Kang YG, Chang CB, Song J, Jeon YT, Kim TK. Use of reduced-dose periarticular injection for pain management in simultaneous bilateral total knee arthroplasty. J Arthroplasty. 2012;27:1731-6.

7. Skeppholm M, Olerud C. Pain from donor site after anterior cervical fusion with bone graft: a prospective randomized study with 12 months of follow-up. Eur Spine J. 2013;22:142-7.

8. Scheerlinck LM, Muradin MS, van der Bilt A, Meijer GJ, Koole R, Van Cann EM. Donor site complications in bone grafting: comparison of iliac crest, calvarial, and mandibular ramus bone. Int J Oral Maxillofac Implants. 2013;28:222-7.

9. Cho SW, Kim DH, Lee GC, Lee SH, Park SH. Comparison between autogenous bone graft and allogenous cancellous bone graft in medial open wedge high tibial osteotomy with 2-Year Follow-up. Knee Surg Relat Res. 2013;25:117-25.

10. Niu YF, An XF, Wu DJ, Xu SG, Zhang CC, Li M. Anatomical reconstruction of donor site after large iliac crest graft harvest with equivalent iliac crest allograft: a prospective controlled study. Eur Rev Med Pharmacol Sci. 2013;17:1951-7.

11. Tanishima T, Yoshimasu N, Ogai M. A technique for prevention of donor site pain associated with harvesting iliac bone grafts. Surg Neurol. 1995;44:131-2.

12. Reuben SS, Vieira P, Faruqi S, Verghis A, Kilaru PA, Maciolek H. Local administration of morphine for analgesia after iliac bone graft harvest. Anesthesiology. 2001;95:390-4.

13. Bojescul JA, Polly DW Jr, Kuklo TR, Allen TW, Wieand KE. Backfill for iliac-crest donor sites: a prospective, randomized study of coralline hydroxyapatite. Am J Orthop (Belle Mead NJ). 2005;34:377-82.

14. Ito M, Abumi K, Moridaira H, Shono Y, Kotani Y, Minami A, Kaneda K. Iliac crest reconstruction with a bioactive ceramic spacer. Eur Spine J. 2005;14:99-102. 